\title{
Internet Compressed Traffic: A Solution for the Explosion of the Internet
}

\author{
Mohammed. A. Al-Shargabi \\ Faculty of Computer Science \& \\ Information Systems \\ Universiti Teknologi Malaysia
}

\author{
Abdul Samad Ismail \\ Faculty of Computer Science \& \\ Information Systems \\ Universiti Teknologi Malaysia
}

\author{
Sevia M.Idrus \\ Faculty of Electrical Engineering \\ Photonic Technology Centre \\ Universiti Teknologi Malaysia
}

\begin{abstract}
The Internet has become a very important communication medium of our daily lives in many fields. People nowadays can study, buy, watch, and obtain more information and more and more services from the Internet. Therefore, the Internet networks have to carry different types of data, such as voice, video, image, and text data. These several types of data grow the Internet traffic up with the needs of providing Quality of Service (QoS) for each type of data. Thus, reducing the size of transmission and storage has become a focal area in the current research, as a solution for the traffic growth. In this paper, a survey of the current literature of the Internet traffics, traffic contract, traffic forms, and its parameters have been introduced. This survey shows the advantages of CBR and VBR traffic that make them in the nearest future two of the most traffic used in the Internet networks.
\end{abstract}

\section{Keywords}

Internet Traffic, CBR, VBR, UBR, and ABR.

\section{INTRODUCTION}

The Internet has become one of the most important communication media in the modern world [1]. It has become a very important catalyst in various areas of life such as economics, education, health, e-governments, social networking, news networks, television and other services that use the Internet to improve its performance.

All these features have attracted more and more people to utilize the benefits of the Internet permanently, causing the number of Internet users to be exponentially increased [2].

Studies have shown a statistically significant increase in the number of Internet users for the last fifteen years, which threatens to get the problem of an explosion of the Internet as shown in Figure 1 [3].

To solve the problem of the explosion of the Internet, the Internet backbone infrastructure must be able to handle a huge amount of traffic, and guarantee the traffic needs.

At the same time, the choice of the Internet traffic structures that will be carried on the Internet backbone infrastructure should be able to reduce both transmission and storage costs $[4,5]$.

In the Internet traffic structures, reducing the size of transmission and storage has become a focal area in the current research, as a solution for the traffic growth $[6,7]$.

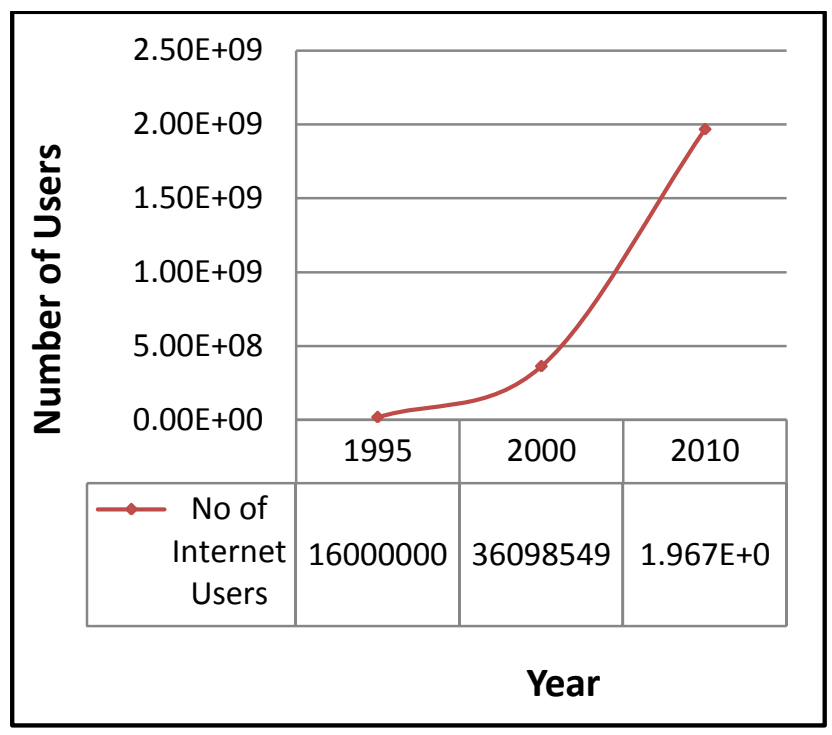

Fig. 1 The number of Internet users for the last fifteen years [3]

\section{INTERNET TRAFFIC}

The Internet networks have to carry different types of data, such as voice, video, image, and text data. Internet traffic can be classified, as shown in Figure 2 into compressed traffic and uncompressed traffic.

Compressed traffic has been proposed as a solution for reducing the size of transmission and storage. Where, the traffic goes through the compression process at the source node and travel in the network in the form of compressed data, which will be decompressed at the destination node. Uncompressed traffic is the normal traffic that travels through the network in the normal form without any type of data compression. Next section will explain the classification and type of Internet traffic.

\subsection{Uncompressed Traffic}

Uncompressed traffic as mentioned before is the normal traffic that travels through the network in the normal form. Uncompressed traffic can be divided into three different categories: sensitive traffic, best- effort traffic, and the undesired traffic. 


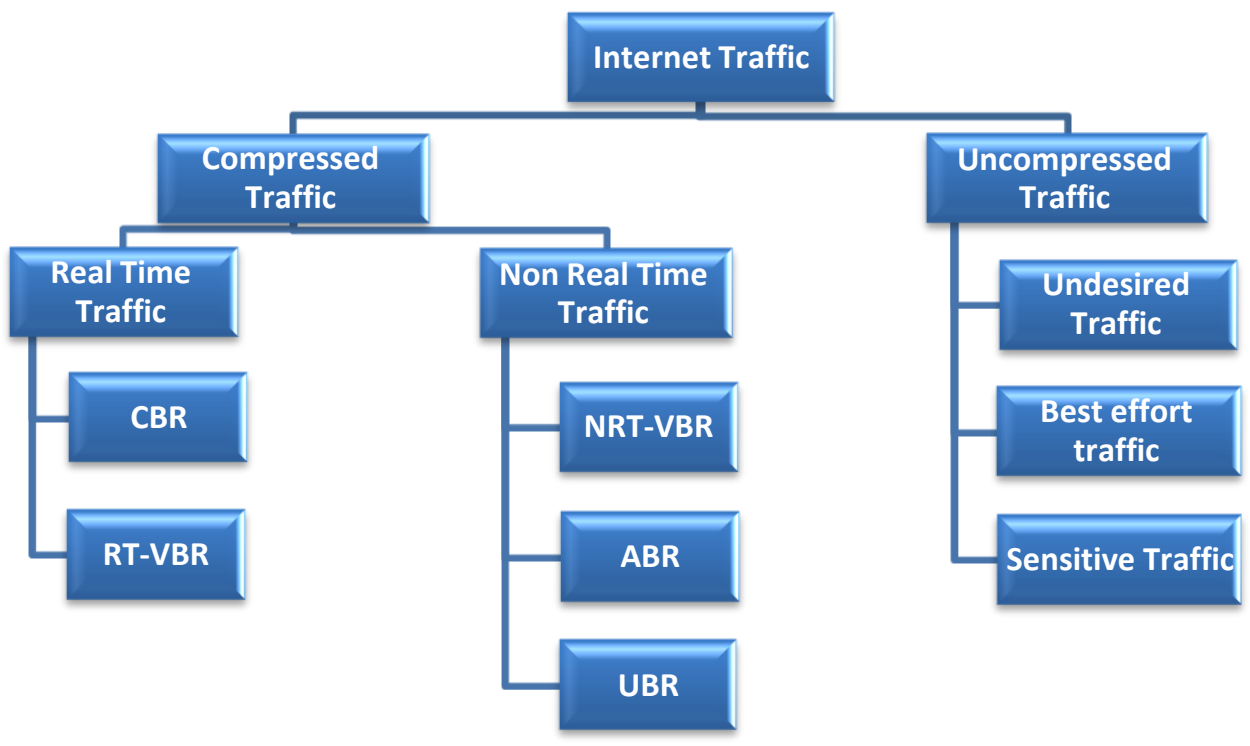

Fig. 2 The Internet traffic classification

\subsubsection{Sensitive traffic}

Sensitive traffic is the traffic transmitted over the network if there is an online interaction between the source and the destination. Consequently, it must be delivered to the destination with a minimum delay, less packets drops, and it has real-time network services. Moreover, the real time traffic such as VoIP, video streaming, and voice and video communication applications are sensitive to the QoS metrics such as latency, packet loss, and jitter. In the real time traffic, the information about the traffic cannot be obtained in advance, so it needs online processing.

\subsubsection{Best-effort traffic}

Best-effort traffic, in contrast, there is no online interaction, which leads to transmit the data over the network with ignoring the QoS metrics. In the best-effort traffic, it is easy to extract the entire traffic information before it is transmitted. Thus, the appropriate network resource allocation is easier to be made. In terms of bandwidth allocation, the best-effort traffic gets the bandwidth left by the sensitive traffic.

\subsubsection{The undesired traffic}

The undesired traffic is created by the delivery of the spam traffic worms, bonnets, and other malicious attacks. In the network configuration, it involves identifying and blocking.

\subsection{Compressed Traffic}

Compressed traffic is the traffic that goes through the compressing process at the source node and travel in the network in the form of compressed data, which will be decompressed at the destination node. Compressed traffic saves the cost of transmission, time, storage, and the bandwidth.

ATM service category [8] is one of the traffic contract concepts that come in the form of compressed traffic. ATM traffic contracts are part of the mechanism by which QoS is ensured. There are four basic types; each one has a set of parameters describing the connection that are Constant Bit Rate (CBR), Variable Bit Rate (VBR) Unspecified Bit Rate (UBR), and Available Bit Rate (ABR).

\subsubsection{Constant Bit Rate (CBR) Traffic}

$\mathrm{CBR}$ is a real time traffic that consume and constant the sending rate, where the traffic sending rate is specified at the Peak Cell Rate (PCR) parameter. CBR is used for connections that need traffic on-time synchronization between the source and destination.

CBR is suitable for the data that the end-systems meed predictable response time and a static amount of bandwidth [9, $10]$.

CBR is useful for multimedia streaming content on limited capacity channels since it is the maximum bit rate that matters, not the average, so CBR would be used to take advantage of all the capacities. CBR would not be the best option to utilize the storage and bandwidth due to it allocate the same bandwidth for complex sections as well as for simple sections. However, CBR provides low-latency traffic with expected delivery description; therefore, it is typically used for circuit switching.

\subsubsection{Variable Bit Rate Traffic}

VBR traffic is one of the loss and delay sensitive traffic, which has become a focal area in the recent research, as a solution for reducing the size of transmission and storage [11-13]. The VBR has been known as an ATM service category that is intended for both the real-time and non-real-time applications [7, 14, 15].

The Real Time-VBR (RT-VBR) service category is used for connections that carry traffic at variable rate, which depends on accurate timing between the source and destination. Real-time VBR connections can be categorized by a Peak Cell Rate (PCR), Sustained Cell Rate (SCR), and Maximum Burst Size (MBS). Packets delay can be specified by the maximum CTD (Cell Transfer Delay) (MaxCTD) parameter.

The Non Real Time-VBR (NRT-VBR) service category is used for connections that carry variable bit rate traffic, which no need for on-time synchronization between the source and destination, but there is a need for an attempt at a guaranteed bandwidth or latency. 
VBR compressed video and audio traffic have been receiving considerable attention due to their compression algorithms that can transmit higher rate during the high activity and low rate during the less activity. The VBR encoding allocates higher rate during the very complex parts of the traffic and lower rate during the smooth parts of the traffic, i.e. silence parts.

\subsubsection{Available Bit Rate}

Available Bit Rate (ABR) service category is intended for the sources having the ability to increase their data rate and use the available bandwidth. ABR is used for connections that transport variable bit rate traffic for which there is no reliance on time synchronization between the traffic source and destination, and for which no required guarantees of bandwidth or latency [16]. ABR provides a best-effort transport service, in which flowcontrol mechanisms are used to adjust the amount of bandwidth available to the traffic originator. The ABR service category is designed primarily for any type of traffic that lacks time sensitivity and expects no guarantees of service.

\subsubsection{Unspecified Bit Rate Traffic}

Unspecified Bit Rate (UBR) service category is intended for the best effort traffic and the non-critical applications that are very tolerant of delay and packets loss. UBR is similar to ABR but there are no flow-control mechanisms to adjust dynamically the amount of bandwidth available to the user [5]. Figure 3 shows the bandwidth partitions with the ATM service categories.

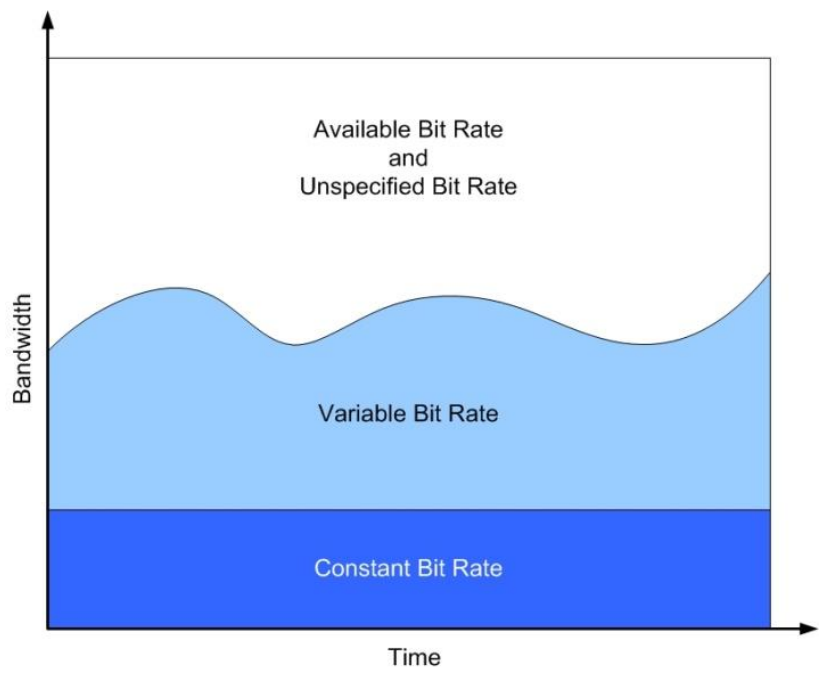

Fig. 3 The ATM service categories

\subsection{Traffic contract}

Traffic contract is an agreement between Internet users and Internet access provider for data exchange specifications and requirements such as the Type of Service $(\mathrm{ToS})$ required, the traffic parameters of each data flow in both directions, and the QoS Parameters requested in each direction. The Internet user application notifies the network about the traffic type that will be transported, and the traffic performance requirements of that traffic $[17,18]$.

\subsubsection{Traffic Parameters}

Traffic parameters are used to configure the traffic formed. There are four main traffic parameters that are: PCR, SCR, MaxCTD, and MBS $[8,15]$.
The PCR is an upper limit of bits of data (in kilobits per second) on the source traffic that can be sent. The SCR is the average of bits of data (in kilobits per second) on the source traffic that can be sent.

The MaxCTD is a parameter that determines the packet maximum delay in the network. Where, the MBS defines the amount of time or the duration at which the source sends at the PCR.

\section{CONCLUSION}

This paper has introduced the advantages of compressed traffic as a solution for reducing the volume of transmission and storage which could a solution for the internet explosion. CBR, VBR, UBR, and ABR are four main traffic types proposed from Asynchronous Transfer Mode (ATM) service category that is come in the form of compressed traffic as traffic contract concepts. ATM traffic contracts are part of the mechanism by which QoS is ensured. CBR and VBR traffic are delay and loss sensitive traffics (real time traffic). CBR is suitable for the data that the end-systems require to predictable response time and a static amount of bandwidth is continuously available for the lifetime of the connection. VBR is an ATM service category that is intended for both the real-time and non-real-time applications. RT-VBR service category is used for connections that transport traffic at variable rate traffic that relies on accurate timing between the traffic source and destination.

\section{REFERENCES}

[1] de Brito Carvalho, T., et al., Key Issues on Future Internet, in New Network Architectures, T. Tronco, Editor. 2010, Springer Berlin / Heidelberg. p. 221-236.

[2] Guo-Qing, Z. and et al., Evolution of the Internet and its cores. New Journal of Physics, 2008. 10(12): p. 123027.

[3] MiniwattsMarketingGroup. WORLD INTERNET USAGE AND POPULATION STATISTICS. 2010 30-June-2010 [cited; Available from: www.internetworldstats.com.

[4] Van der Auwera, G., P. David, and M. Reisslein, Traffic characteristics of H.264/AVC variable bit rate video. Communications Magazine, IEEE, 2008. 46(11): p. 164174.

[5] Andrikopoulos, I., et al., Providing rate guarantees for internet application traffic across ATM networks. Communications Surveys \& Tutorials, IEEE, 1999. 2(3): p. 2-13.

[6] [Beran, J., et al., Long-range dependence in variable-bitrate video traffic. Communications, IEEE Transactions on, 1995. 43(234): p. 1566-1579.

[7] Spohn., D.E.M.a.D.L., ATM Theory and Applications 1st ed. 1998: McGraw-Hill Professional.

[8] ATMFORUM, UNI Signaling 4.0 1996.

[9] Ahmadi, H., et al., NBBS traffic management overview. IBM Systems Journal, 1995. 34(4): p. 604-628.

[10] Ghiassi-Farrokhfal, Y. and J. Liebeherr, Output Characterization of Constant Bit Rate Traffic in FIFO Networks. Ieee Communications Letters, 2009. 13(8): p. 618-620. 
[11] Veiro, B., Traffic Measurement on Variable Bit Rate (Vbr) Sources with Application to Charging Principles. Computer Networks and Isdn Systems, 1990. 20(1-5): p. 435-445.

[12] Van der Auwera, G., P.T. David, and M. Reisslein, Traffic Characteristics of H.264/AVC Variable Bit Rate Video. Ieee Communications Magazine, 2008. 46(11): p. 164-174.

[13] Mao, G.Q. and H.B. Liu, Real time variable bit rate video traffic prediction. International Journal of Communication Systems, 2007. 20(4): p. 491-505.

[14] Roberts, J.W., Variable-bit-rate traffic control in B-ISDN. Communications Magazine, IEEE, 1991. 29(9): p. 50-56.
[15] ATMForum, UNI Signaling 4.0 1999.

[16] Bonomi, F. and K.W. Fendick, The rate-based flow control framework for the available bit rate ATM service. Network, IEEE, 1995. 9(2): p. 25-39.

[17] DaSilva, L.A., Pricing for QoS-enabled networks: A survey. Communications Surveys \& Tutorials, IEEE, 2000. 3(2): p. 2-8.

[18] Roberts, J.W., Internet traffic, QoS, and pricing. Proceedings of the IEEE, 2004. 92(9): p. 1389-1399. 